\title{
P34 24-Hour Ambulatory Brachial Versus Aortic Systolic Blood Pressure: Relationship with Left Ventricular Mass Significantly Differs. Pooled Results from the International 24 Hour Aortic Blood Pressure Consortium (i24ABC)
}

\author{
Siegfried Wassertheurer ${ }^{1, *}$, Athanase Protogerou ${ }^{2}$, James Sharman ${ }^{3}$, Enrique Rodilla Sala ${ }^{4}$, Piotr Jankowski ${ }^{5}$, \\ Maria Lorenza Muiesan ${ }^{6}$, Cristina Giannattasio ${ }^{7}$, Bernhard Hametner ${ }^{8}$, Jose Maria Pascual ${ }^{4}$, Robert Zweiker \\ Antonio Argyris ${ }^{2}$, Anna Paini ${ }^{6}$, Ian Wilkinson ${ }^{10}$, Danuta Czarnecka ${ }^{5}$, Massimo Salvetti ${ }^{6}$, Alessandro Maloberti ${ }^{7}$, \\ Carmel McEniery ${ }^{10}$, Yan $\mathrm{Li}^{11}$, Janos Nemcsik ${ }^{12}$, Giacomo Pucci ${ }^{13}$, Cornelia Ablasser ${ }^{9}$, Jacques Blacher ${ }^{14}$, \\ Alexandre Valleé ${ }^{14}$, Alejandro de la Sierra ${ }^{15}$, Yi Zhang ${ }^{16}$, Hongwei $\mathrm{Ji}^{16}$, Barry McDonnell ${ }^{17}$, Marco Mota ${ }^{18}$, \\ Annelise Paiva ${ }^{18}$, Andrea Brandao ${ }^{18}$, Thomas Weber ${ }^{19}$ \\ ${ }^{1}$ AIT Austrian Institute of Technology, Vienna, Austria \\ ${ }^{2}$ Cardiovascular Prevention and Research Unit, Department of Pathophysiology, Medical School, Laiko Hospital, National and \\ Kapodistrian University of Athens, Greece \\ ${ }^{3}$ Menzies Institute for Medical Research, College of Health and Medicine, University of Tasmania, Australia \\ ${ }^{4}$ Department of Medicina Interna, Hospital de Sagunto, Universidad Cardenal Herrera-CEU, CEU Universities, Valencia, Spain \\ ${ }^{5}$ Department of Cardiology, Interventional Electrocardiology and Hypertension, Jagiellonian University, Medical College, Krakow, Poland \\ ${ }^{6}$ Department of Clinical and Experimental Sciences, University of Brescia, Italy \\ ${ }^{7}$ Cardiology IV, 'A. De Gasperis' Department, ASTT Ospedale Niguarda Ca' Granda, School of Medicine and Surgery Department, \\ Milano-Bicocca University, Milan, Italy \\ ${ }^{8}$ Center for Health and Bioresources, Department of Health and Environment, Austrian Institute of Technology, Vienna, Austria \\ ${ }^{9}$ Cardiology Department, Medical University Graz, Austria \\ ${ }^{10}$ Division of Experimental Medicine and Immunotherapeutics, University of Cambridge, UK \\ ${ }^{11}$ Ruijin Hospital, Shanghai Jiaotong University School of Medicine \\ ${ }^{12}$ Semmelweis University Budapest, Hungary \\ ${ }^{13}$ Unit of Internal Medicine, Terni University Hospital, Department of Medicine, University of Perugia, Italy \\ ${ }^{14}$ Diagnosis and Therapeutic Center, Hotel Dieu; AP-HP; University Paris Descartes, France \\ ${ }^{15}$ Hospital Mutua Terrassa, University of Barcelona, Spain \\ ${ }^{16}$ Department of Cardiology, Shanghai Tenth People's Hospital, Tongji University School of Medicine, China \\ ${ }^{17}$ Cardiff Metropolitan University, UK \\ ${ }^{18}$ Centro Universitário Cesmac/Hospital do Coração de Alagoas, Maceió Brazil \\ ${ }^{19}$ Klinikum Wels-Grieskirchen, Kardiologie, Wels, Austria
}

\begin{abstract}
Background: There is evidence of a closer relation between 24 hour aortic systolic blood pressure (aSBP) and left ventricular mass (LVM) compared with 24 hour brachial SBP. However, sample sizes are relatively small and there is some inconsistency in findings. We sought to address this by pooling data from 16 centers in Asia, Europe and Latin America to determine the relationship between LVM and brachial office, as well as brachial and aortic 24 hour ambulatory SBP.

Methods: In all centers, brachial and aortic SBP was measured with the same validated oscillometric device, using a transfer function for aortic pressure, and mean/diastolic pressure calibration. LVM was determined by echocardiography.

Results: We studied 2092 participants (972 women) with a mean age of 52 years. Mean brachial office BP was $137 / 84 \mathrm{mmHg}$ and mean 24 hour bSBP and aSBP was $126[118 ; 134]$ and $131[120 ; 137] \mathrm{mm} \mathrm{Hg}$, respectively. Mean LVM indexed to body surface area was $88.4 \mathrm{~g} / \mathrm{m}^{2}$, and $27.2 \%$ of participants had left ventricular hypertrophy (LVH). The correlation coefficients between LVM and brachial office SBP, 24 hour bSBP, and 24 hour aSBP were $0.24,0.35$, and 0.43 , respectively $(p<0.001$ for differences). The areas under the curve for prediction of LVH were $0.62,0.67$, and 0.70 for brachial office SBP, 24 hour bSBP, and 24 hour aSBP, respectively ( $p<0.001$ for differences).

Conclusion: In this pooled analysis of international data, we demonstrate that aortic ambulatory SBP, measured with an oscillometric cuff, shows a significantly closer association with hypertensive cardiac organ damage (left ventricular mass and hypertrophy) than brachial office/brachial ambulatory systolic blood pressure.
\end{abstract}

(c) 2019 Association for Research into Arterial Structure and Physiology. Publishing services by Atlantis Press International B.V. This is an open access article distributed under the CC BY-NC 4.0 license (http://creativecommons.org/licenses/by-nc/4.0/). 